\title{
Design of a Rescue Robot Assist at Fire Disaster
}

\author{
A. Rama Krishna \\ Assistant Professor \\ Dept. of E.C.M \\ K.L.University, Guntur
}

\author{
B. Bhanu Prakash Sarma \\ B-Tech IV/IV \\ Dept. of E.C.M \\ K.L.University, Guntur
}

\author{
G. Sowmya Bala \\ PG Scholar \\ Dept. of E.C.M \\ K.L.University, Guntur
}

\author{
Gokul Sai Alla \\ B-Tech IV/IV \\ Dept. of E.C.M \\ K.L.University, Guntur
}

\author{
A. S. N. Chakravarthy, PhD. \\ Professor \\ Dept. of E.C.M \\ K.L.University, Guntur
}

\begin{abstract}
The present manuscript comes with an innovative thought of designing a rescue robot, which aims in aiding human caught on fire. The proposed robotic system will rescue into the fire and detect the human (being alive). The fire protective object detection sensor is used, as it has the ability to reserve its features even in the environment surrounded with smoke and high degree centigrade temperature (due to the fire explored). The body of the robot is made-up of a metal that protect its internal components. Arm 7 Micro controller to provide control and co-ordination between every component is adopted. The information regarding the victim can be communicated with the aid of mobile ad-hoc networks. Wireless communication system assists the robot by enabling the communication between the victim and the RT (Rescue Team). Mobile Ad Hoc Networking (MANET) based wireless communication algorithm has been used to provide the back and forth message transmission. The robot can send the feedback to an operator generally located at certain distance, which can guide the rescue team by providing in time information that saves the lives of victims. The entire robot deign is fire and heat protective so that it can easily enter into the regions where a rescuer cannot and can communicate to the RT (Rescue Team) by sending feedback regarding those who trapped on fire.
\end{abstract}

\section{Keywords}

MANET, ARM7 microcontroller, Ultra Sonic Object Detection Sensor, wireless communication,

\section{INTRODUCTION}

Disasters may be natural or man-made, which ends with a vast loss of valuable lives. These disasters may be of earthquakes, fire accidents, heavy rains, explosion of heavy solids or due to chemical reactions, and all. Whatever may be the form, it ends by taking the valuable lives of many innocents. Rescue operations in such situations are a risky and deadly process that may saves someone's life but not everyone's. Due to the environmental conditions and some other disturbances, a rescuer cannot enter into the most critical areas where there is a hope of someone to be alive.

This is a most common situation in disaster caused out of fire accidents. In the fire accidents, it is highly risk process for a RT (Rescue Team) to save the lives and even to find and communicate with those who captured in it. Currently, in order to overcome these problems and to survive the victims, the robotic technology is on pinnacle and is the most commonly adopted method. With the aid of the most advanced technology and the equipment's available at present a rescue robot is designed that can help the RT (Rescue Team) by entering into the areas where the RT unable to go.

This paper mainly focus on designing such a robot that can act as a rescuer and enter into even critical areas, it searches and send the information about the victims if any in that area. The information will be sent to the RT through the most prominent wireless communication technology that has been adopted and has a vital role as it locates the human and the robot [4]. The robot sends the information to the RT there on the site (at rescue operation). As the RT is nearer to the accident zone, the distance for information to travel is around few meters only. Mobile Ad-hoc Network (MANET) is a way of wireless communication that is very efficient for communicating with in short distances [4]. A group of robots can enter into the rescue field and with the help of one other, they form a communication network that is a mobile ad-hoc communication network where each robot themselves act as a communication network node. The emergence of the Mobile Ad Hoc Networking (MANET) technology support selforganized wireless interconnection of communication devices that would either extend or operate in concert with the wired networking communications or, possibly, advance to autonomous networks [4]. In general, a sensor network is composed of a large number of small sensor nodes, which are closely deployed at random positions inside the area in which an event is being monitored. The sensor node consumes power for sensing, communicating and data processing. More energy is required for data communication than any other process.

In short, the wireless sensor networks hold great promise as an enabling technology for a variety of applications, including data collection and event detection. The basis for sensor network has been constituted with this wireless ad hoc networking technique. However, the special constraints imposed by the unique characteristics of sensing devices, and by the application requirements, make many of the solutions designed for multihop wireless networks (generally) not suitable for sensor networks.

As the robot has to rescue into the fire, the internal parts of the robot has to be fireproofed and have to prevent the internal components from being damaged. Therefore, the choice of a metal with which the robot is shielded is an 
important task. The robot has to detect the human with the help of an object-detecting sensor. Human sensing is a challenging endeavor for a variety of reasons [9]. These challenges are due to the obstacles caused due to some reasons such as:

- Appearance variability and unpredictability

- Environmental variations

- Sensing noise signals or unwanted signals

Sensor has to detect the object in a severe environment where there is a high temperature and of course, the surroundings are completely covered with heavy smoke. Some times there maybe a possibility of explosions due to chemical reactions and all. Therefore, the sensor has to detect in any of this situations. FW Series objecting detecting ultrasonic sensors [2] with maximum detection range of 39.37". It is used in automotive manufacturing applications, the sensors feature target height display and provide stable detection in severe environments via an external shift function [2]. Another type of the sensors used to detect the objects are PIL ultrasonic object detecting sensors that are available with sensing ranges of $11.8 \mathrm{~mm}$ to $6000 \mathrm{~mm}$ [2]. The sensors are often used to detect shiny objects, or objects found in foggy or particle-laden air environments [9]. The robot designed is considerably smaller than a human is and they operate closer to the floor where the smoke can be expected to be less dense. Even there is a possibility of low temperature nearer to the floor, which can be an additional advantage for the robot design [9]. Regarding the materials that has been used to shield the entire robot is briefly explained in the Literature Survey and this is followed by the design process and message transmission process. This is finally ended with a conclusion.

\section{LITERATURE SURVEY}

\subsection{Choice of a protective shield}

As mentioned in the introduction the robot has to be shielded with an eminent and fire resistive metal body. It should be able to protect its internal components like controllers, sensors, battery chargers and all other devices inbuilt with in a robot.

In general, metals are classified as ferrous or nonferrous. A ferrous metal has iron as its main element. Nonferrous metals include many metals that are used mainly for metal plating or as alloying elements, such as tin, zinc, silver, and gold [7]. However, the focus is only on the metals used in the manufacture of parts, such as aluminum, magnesium, titanium, nickel, copper, and tin alloys. The internal reactions of a metal to external forces are known as mechanical properties [7]. The mechanical properties are directly related to each other. A change in one property usually causes a change in one or more additional properties. The melting point of the meal is the important property in this design process as the metal so far used should be able to withstand with in certain temperature ranges [7]. Here are some metals with their melting point ranges. It is important to consider their melting point ranges [5] up to that the metal can withstand.
Table 1 Metals and their Melting point ranges.

\begin{tabular}{|c|c|c|}
\hline \multirow[t]{2}{*}{ Metal } & \multicolumn{2}{|c|}{ Melting Point } \\
\hline & $\left({ }^{\circ} \mathrm{C}\right)$ & $\left({ }^{\mathbf{0}} \mathbf{F}\right)$ \\
\hline Beryllium & 1285 & 2345 \\
\hline Cast Iron, gray & $1175-1290$ & $2150-2360$ \\
\hline Chromium & 1860 & 3380 \\
\hline Cobalt & 1495 & 2723 \\
\hline Copper & 1084 & 1983 \\
\hline Cupronickel & $1170-1240$ & $2140-2260$ \\
\hline Gold & 1063 & 1945 \\
\hline Hastelloy C & $1320-1350$ & $2410-2460$ \\
\hline Inconel & $1390-1425$ & $2540-2600$ \\
\hline Incoloy & $1390-1425$ & $2540-2600$ \\
\hline Iridium & 2450 & 4440 \\
\hline Iron & 1536 & 2797 \\
\hline Manganese & 1244 & 2271 \\
\hline Molybdenum & 2620 & 4750 \\
\hline Monel & $1300-1350$ & $2370-2460$ \\
\hline Nickel & 1453 & 2647 \\
\hline Niobium (Columbium) & 2470 & 4473 \\
\hline Osmium & 3025 & 5477 \\
\hline Platinum & 1770 & 3220 \\
\hline Rhodium & 1965 & 3569 \\
\hline Silicon & 1411 & 2572 \\
\hline Carbon Steel & $1425-1540$ & $2600-2800$ \\
\hline Stainless Steel & 1510 & 2750 \\
\hline Tantalum & 2980 & 5400 \\
\hline Thorium & 1750 & 3180 \\
\hline Titanium & 1670 & 3040 \\
\hline Tungsten & 3400 & 6150 \\
\hline Uranium & 1132 & 2070 \\
\hline Vanadium & 1900 & 3450 \\
\hline Zirconium & 1854 & 3369 \\
\hline
\end{tabular}

The robot body is covered with an appropriate metal in a proper way that it should not cause any damage to the internal body parts/components. The metal so far used will provide a fireproof. The choice of the metal to be used will depends on the severity of the fire at the accident zone. So far it is not possible to measure the severity, so a proximity value is considered and within that range, an appropriate metal is been chosen. The cost of the metal is also an important factor. Due to some minute mistakes, there may be chances to cause damage to the robot. Once if the cost is with in affordable range, the damage severity does not matter for. As the robots size is smaller and is closer to the ground/floor where the fire severity will be less compared to the other regions, the chances of the damage will be less. Therefore, these are some factors regarding the chances to cause damage and that does not cause damage.

- $\quad$ Size of the robot designed must be small enough.

- Choice of the metal must be cost effective.

- The melting point range of the metal should be high such that it averts the mutilation to the other components.

It is clearly mentioned in the introduction about the challenges that a sensor has to face while detecting a human being mainly in disaster environments. In this literature, survey few problems are listed out that can swerve a sensor from 
detecting a victim. This may result out due to the improper gathering of information, loss of some visibility mainly due to smoke in case of fire accidents, due to some obstacles like walls, doors, heavy pieces of rock or bricks and a like. The sensors are somehow prone to shot noise due to statistical fluctuations in the particle arrival rates [9]. There are some other kinds of noises that should be alleviated through the hardware design considerations of a sensor.

In the real-world scenarios, there must be some common sources of errors due to the sudden changes in the surrounding environment. These common sources depend on the fire severity and the smoke that slowly surrounds the whole area, and due to the lighting variations, shadows, air conditioning, and so on. The sensor signals may be distracted due to the unwanted signals from the surroundings like noise caused by loud sounds, explosions, so on. This may sometimes leave the robot in a cessation state, as it cannot take a proper decision in time. The sensor information is an important factor for the robot to take the timely decisions. The sensor may detect not only the information regarding the human but also his /her condition. It is important, as in most of the fire accidents the death rate increases due to the heavy smoke as it spreads faster than the fire particles. This heavy smoke will immediately swoons the persons inside the room. Therefore, the sensor will be able to see whether the person is alive or not. This can be done by sensing the oxygen levels of the person and the breath rate. It is important that all this information should be processed within seconds' gap, as the time is a major factor in such situations.

As mentioned above at some fire accidents there is a possibility of heavy explosions due to the chemical gases or due to some perilous active chemicals undergoes some reactions resulting in heavy explosions. Therefore, there is a need to monitor this factor too and in a pre-explosive concentration. The chemical sensors are used in such environments $[1,3]$. This chemical sensor requires very little power to operate $[1,3]$. There are different types of commercially available chemical sensors. Chemical sensors are used for many purposes, such as environmental hazard screening, explosives detection, product characterization, and medical testing [6].

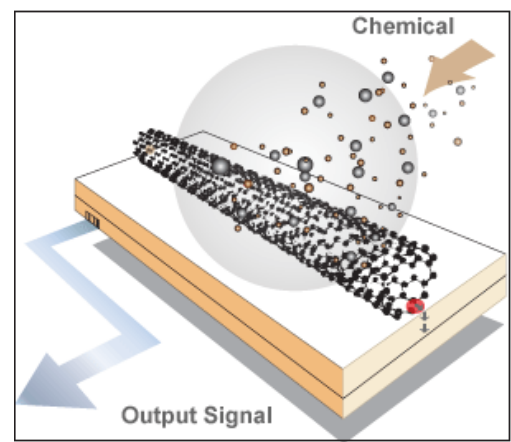

Fig 1: Chemical Sensor detection process [1]

Of them, the sensor built of Metal-oxide semiconductor and home built quartz crystal microbalance sensors are the most apt [6]. These sensors are applied to detect low and high concentrations of the explosions. In general, the electrochemical sensors are widely used in portable instruments that contain multiple sensors $[1,3]$. The life expectancy of the sensor is highly dependent on the environmental contaminants, temperature, and humidity to which it is exposed [3].

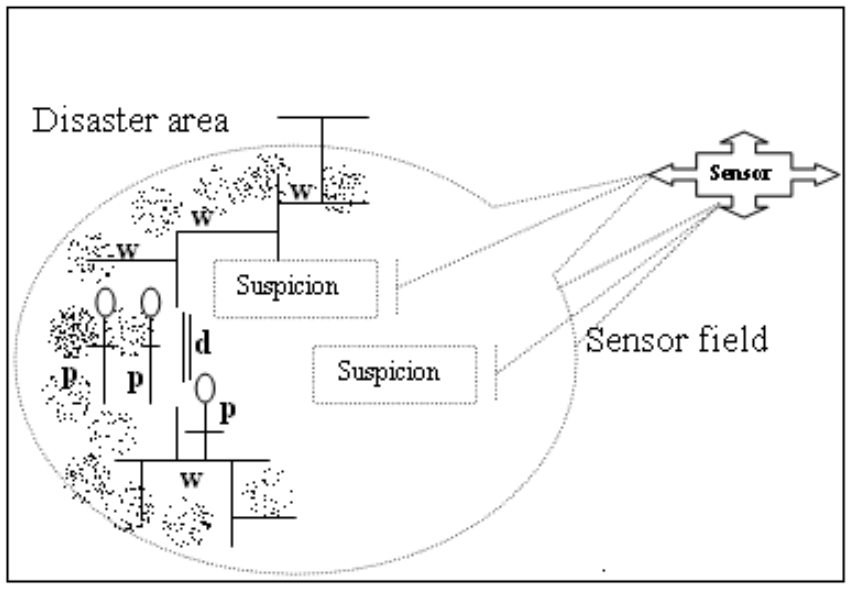

Fig 2: Sensor Field detecting the victims captured on fire in the disaster area.

p-people, d-door, w-wall

As shown in the figure 1 the sensor within the sensor field starts to detect the human that are caught on fire in a disaster area. The sensor gets a suspicion signal initially and then collects the information regarding the person. The sensor is able to detect the person behind the wall. As mentioned in the introduction the FW Series objecting detecting ultrasonic sensors are able to detect the object even in the severe environmental conditions [2]. Some sensors like the infrared camera (a visible sensor), pyroelectric sensors detect the human based on the heat conditions. They detect humans only if the human or the sensor is moving [10]. In a disaster area, it is very hard to sort a human sound like a shout. On the other hand, at rare conditions, there may be a possibility to find a survivor by stopping all activity to pay attention to a shouting person [8]. It is possible to hear some people in this condition. In such cases, the microphone is suitable, which is a low cost sensor $[6,8]$. However, it is not very easy to interface to process its data.

\section{DESIGN PROCESS}

The robot design consist of a microcontroller that co-ordinates the control operations between the different parts like sensors, motors and so on, that are being used in its design. Three types of sensors are used for detecting the human (victim). They are

1. FW series and PIL Ultra sonic object detection sensor.

2. MOS chemical sensor

3. Camera (Visibility Sensor)

The first sensor is adopted for detecting objects nothing but the humans (Victims). The features of this sensor are mentioned clearly in the introduction part. As specified in the literature survey a chemical sensor is required to detect the explosions if any due to the active/inactive reactions. It is important to have a co-ordination between the two sensors. Once the robot enters into the accidental zone, the sensor gets activated immediately it sends an active message to the micro controller. The micro controller now starts to perform the control operations and the memory is set to its initial value or initial state. This active message acts as a reset signal to the controller. Now the USOD sensor is on its work of searching for a target (Victim). This USOD sensor will consecutively activates the visibility sensor, which is initially in an inactive state, once if it finds any suspicious object in that zone. 


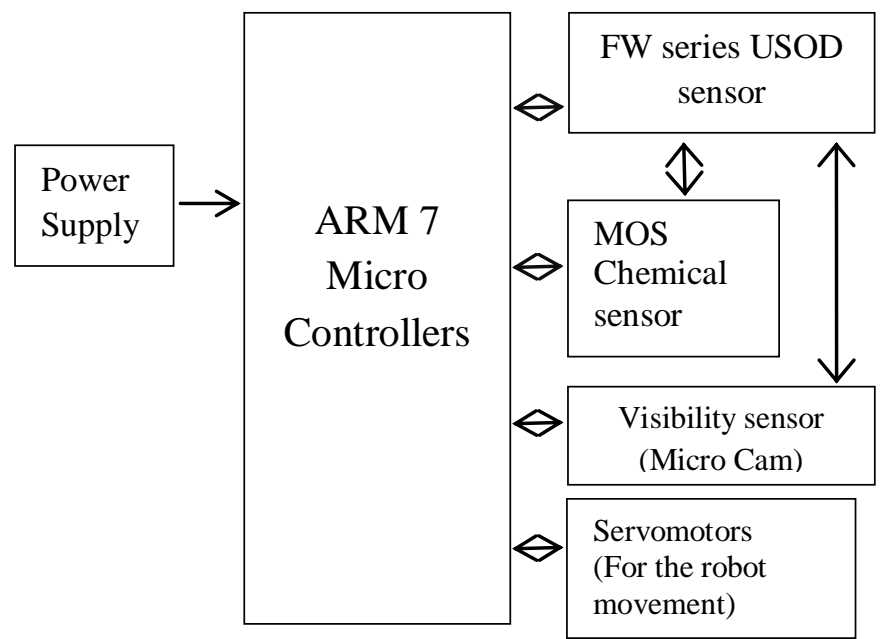

Fig 3: Micro Controller and its Peripherals

The object detection sensor has to filter out the things effectively in order to find its target (Victim) efficiently. The micro controller is used to co-ordinate the control operations between the internal components. This controller monitors the sensors' functioning. The sensors' in turn have to work in consequent of the other. The visibility sensor (or simply camera), gets activated by the USOD sensor. The active signals from the USOD sensor consist of the information collected from the surrounding that undergoes a filtering operation to extract the valid information. Depending upon the victim body movements and some other signals, the sensor detects and locates the victim by considering these features i.e. based on the movement and signals from the victims. The camera is used to identify the appropriate vision of the victim. The controller and the object-detecting sensor receive the information from this sensor. A filtering operation is performed on the input received whose output is verified with the data stored in the object-detecting sensor.

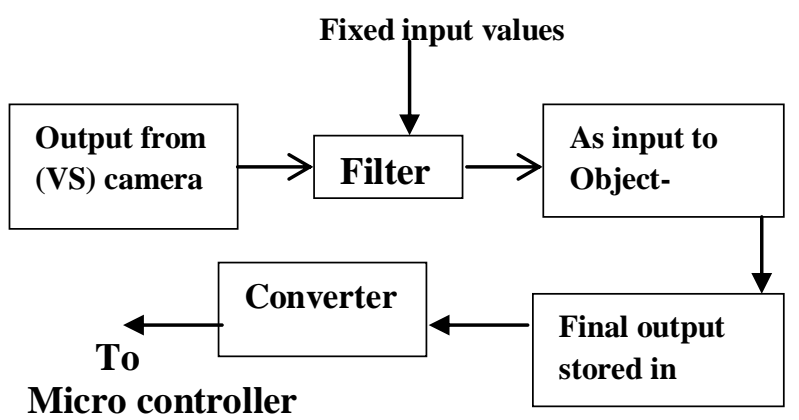

Fig 4: Internal operation of information received from sensor by Micro Controller

As shown in the figure 4 the unwanted signals are subtracted through a filter operation. A fixed input values are assigned initially, according to which the desired values are assigned as input to object-detecting sensor unit. Now the information with in the sensor unit undergoes a verification process with that actually programmed in order to detect the human in such a severe environment. Apart from that, this information is stored in the controllers' memory unit. After a successful transmission, the controller resets its memory unit to store new information. The message transmission is done through the Mobile Ad Hoc Networking algorithm, which is clearly explained in the later section. A combination of CopperCopper coated fire protective shield is used to prevent a sort of device failure due to the heat out of fire. The external body of the robot is covered with this guard. Even a portable coolant machine will be inserted provided if the controller is unable to withhold with in that temperature range.

\section{MESSAGE TRANSMISSION ALGORITHM}

The information collected by the sensor first stores in the memory unit of the controller. This can then send to RT with in the surroundings. The distance for this is short and has to reach to every member of the RT to avoid loss of lives and this can be done with a Mobile Ad-hoc networking algorithm. It is a wireless communication system where each node in the network becomes active every time and sends the information to the other node. Mobile ad hoc networks (MANETs) signify complex distributed systems that encompass wireless mobile nodes that can freely and dynamically self-organize into arbitrary and short-term, "ad-hoc" network topologies, allowing people and devices to seamlessly inter-network in areas where there is non pre-existing communication, e.g., disaster revival surroundings [4].

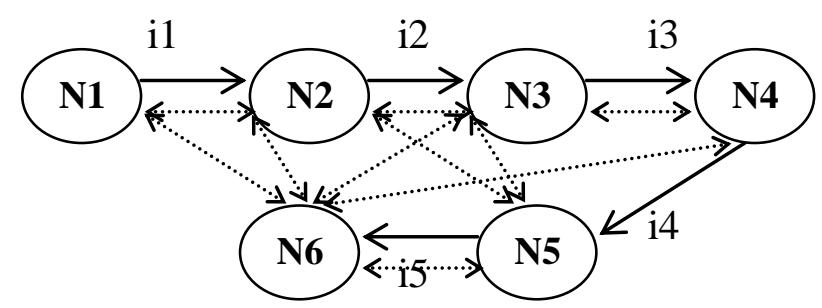

Fig 5: Message Transmission between 6- nodes.

Figure 5 shows a message i transmitted from the node N1 to the node $\mathrm{N} 2$ and it follows the remaining nodes in order to reach its destination. The message chooses the path that is short enough and should reach in short span of time. As time is the main factor to be considered, each node should be active and receive message from every node. The node should be able to keep the information for (n-1) seconds of time where $n$ is the rate at which the message is transmitted.

The message routing depends upon the Link State Routing protocol. It had an advantage over its earlier kind, i.e. Distance Vector based routing protocols, which does not rely for routing only on the shortest path between nodes, but also on the most reliable link (that is least likely to fail) and also the highest quality link (providing least number of packet losses) $[\mathrm{g}]$.

Every node that is at a certain distance near to sender node receives the information send by the robot. Suppose the node 3 is nearer to node1 then it receives the message first and if node 5 is next to node 3 then the message will be directed to it. This is because the nodes are not stationary as each member of the RT (Rescue Team) act as an individual node and are mobile. The information shows the location of the human (victim) it detects. This is sent to every node.

The RT consists of a sensor and a location indicator with in the suit they are provided with, which can act as a node by itself. If more than two robots enter the field then they form a communication network considering each as an individual node and by applying the Link State Routing Protocols the message transmission will be carried out. The information regarding the location of the target victim can be shown in the indicator. 


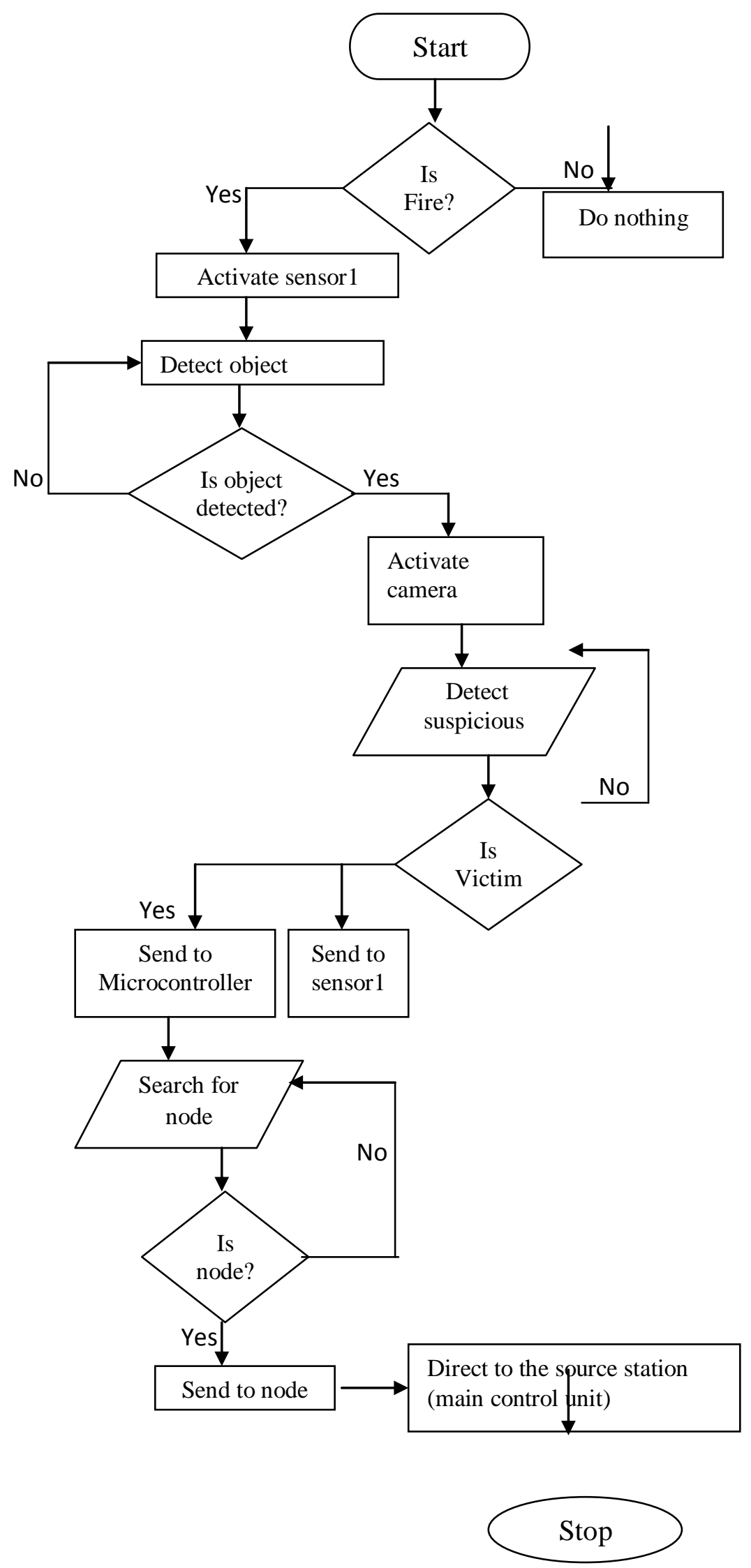

Fig 6: Flow Graph representation of the detection and communication system 


\subsection{Flow Graph Representation of the entire system}

The figure 6 shows the flow graph representation of the entire design process which primarily shows the object/human detection process using sensors and the communication system between the nodes.

These are the steps followed for detecting the object and conveying the information.

\section{Start}

2. Is fire?

3. Activate USOD sensor

4. Is object detected?

5. Set camera in an active mode

6. Is the suspicious a Victim?

7. Send the information to USOD

8. Convert information and send to the microcontroller

9. Micro controller sends the information to the other nodes i.e. RT (Rescue Team) members.

10. Direct to the source station i.e. main control unit.

An RT Suit is provided with the rescue team that consist of a sensor which can act as a network node and helps in forming a communication network.

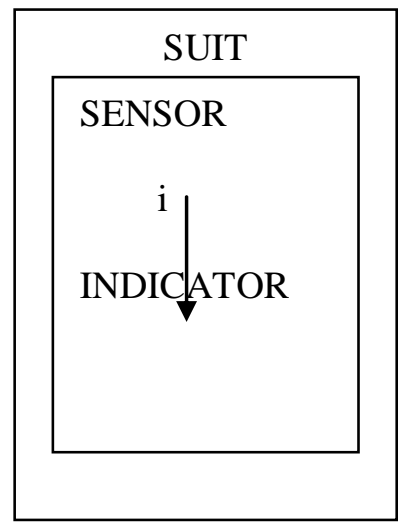

Fig 7: RT suit

The RT suit with the sensor and the indicator is as shown in the figure 7 where the message is represented as i. The location identification system is as shown in the figure 8 . The variable $h$ represents the victim location, the variable $\mathrm{s}$ indicates the suspicious object and the variable $\mathrm{i}$ represents the information regarding the target (Victim).

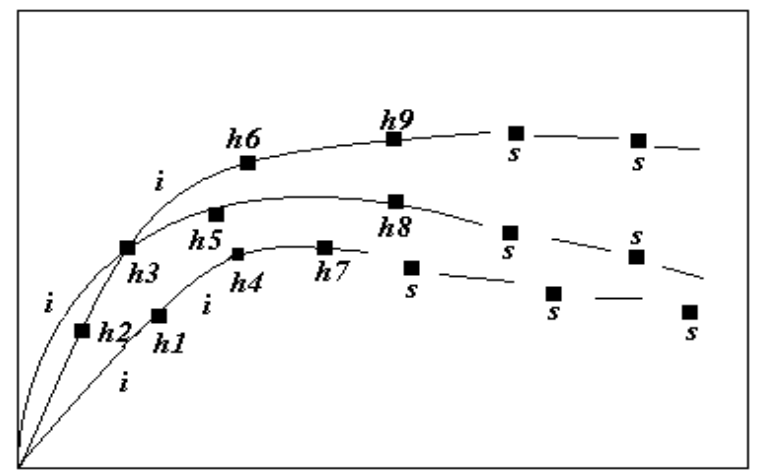

Fig 8: Indicator with in the RT suit (Locating the Victims) The figure 8 above is the information collected by the robot at a zone say Z1.The persons are shown in the form of dots and are indicated as h1, h2, h3 ...h9. The other suspicious objects are also located and are represented by s and the path to reach the victim with detail information about the position is shown well. The victims are located in the order in which the sensor detected i.e. $\mathrm{h} 1$ is detected and located first and then $\mathrm{h} 2$ and so on.

\section{CONCLUSION}

The design process of a rescue robot is explained in this paper. Each aspect for detecting the human in a disaster environment considering a fire accident is explained clearly. The design process covers the communication system and the sensors for detecting the object in a hazard environment. The brief description of the sensor type and the metal body to protect the robot from the heavy fire and smoke is given in the literature survey. The robot designed is aimed to work efficiently and effectively in such severe environments. An efficient wireless communication system is specified in this paper. Mobile Ad-Hoc Networking algorithm has been used that provides a well routed message transmission protocols, which is an important task that locates the human (Victims) and conveys the information regarding the location along with the path that is been traced on an indicator and finally reaches the source station i.e. the main control unit.

\section{FUTURE SCOPE}

The communication algorithm so far used will transmits the information effectively but there may be a chance of error added up with the message signal while sending and receiving the information. Even better algorithm is applied to reduce this kind of transmission errors and to provide more efficient signal transmission.

\section{REFERENCES}

[1]. "ELECTRO CHEMICAL SENSORS" - Bibliography Page: 27-35.

[2]. Http://industrial sensor resource .com / category / ultrasonic - sensors / objection - detection - ultrasonic - sensors.html on 14-4-12

[3]. Http: // www. Nano integris. com / en / chemical sensors on 14-04-12

[4]. Imrich Chlamtac, Marco Conti, Jennifer J.-N. Liu, "Mobile ad hoc networking: imperatives and challenges", Ad Hoc Networks 1www. elsevier. com / locate / adhoc 2003, pg: 13-64.

[5]. "Metals- Melting Temperatures", http://www. engineering toolbox. com / melting- temperaturemetals-d_860.html, on 14-04-12.

[6]. Prabal Dutta, Mike Grimmer, Anish Arora, Steven Bibyk, and David Culler, "Design of a Wireless Sensor Network Platform for Detecting Rare, Random, and Ephemeral Events" in http :// www. cs . berkeley. edu/ prabal/projects/xsm on 2006.

[7]. "PROPERTIES, IDENTIFICATION, AND HEAT TREATMENT OF METALS GENERAL" Bibliography TC 9-524 pages 2-14

[8]. Richard R. Leach, "Cooperative Discrimination Sensor: Detecting and Tracking Human Activity", Fy05 Engineering Research and Technology Report, pg: 80-81.

[9]. Steve Burion, "Human Detection for Robotic Urban Search and Rescue" published on 26-02-2004, Institute De Production Robotique (IPR) LSRO2 - VRAIGroup Diploma Work 2003/2004 pg: 7-61

[10]. Thiago Teixeira Gershon Dublon Andreas Savvidesacm, "A Survey of Human-Sensing: Methods for Detecting Presence, Count, Location, Track, and Identity" in Computing Surveys, Vol. V, No. N, 20YY, Pages 1-35. 